\title{
Use of Programming in SPSS
}

\author{
Halgare Nanasaheb \\ Mahadev \\ Research Scholar Kalinga \\ University \\ Naya Raipur, Raipur (CG)
}

\author{
Ali Akbar Bagwan, PhD \\ Research Supervisor \\ Kalinga University \\ Chhattisgarh, India
}

\author{
D. V. Biradar \\ Head Department of \\ InformationTechnology \\ M.S.Bidve Engineering \\ College, Latur
}

\begin{abstract}
Never done programming? Afraid of or not interested in learning software code and coding? In SPSS, you can use program without knowing how to program or write code! As you go through this paper the meaning of this will become clear. SPSS has two programming languages:

- Script. This language is used mainly for working on output tables and charts. Section 1teaches how to use Scripts.

- Syntax. This language is used for programming SPSS procedures. It is the more important language. Section 2 teaches how to use Syntax.
\end{abstract}

\section{General Terms}

Script, Syntax et. al.

\section{Keywords}

Program, Script Editor, SPSS output Navigator.

\section{INTRODUCTION}

Scripts are programs that provide tools that enable the saving of time in working on output tables, charts, etc. To use Scripts no need to learn programming-- you can use Scripts written by others. Some scripts are supplied with SPSS and were installed on your computer when you installed SPSS .Most Scripts work on SPSS output. So, to learn how to use Scripts, open an output file. (Or use the one supplied with this document-- it is shown in the next picture).

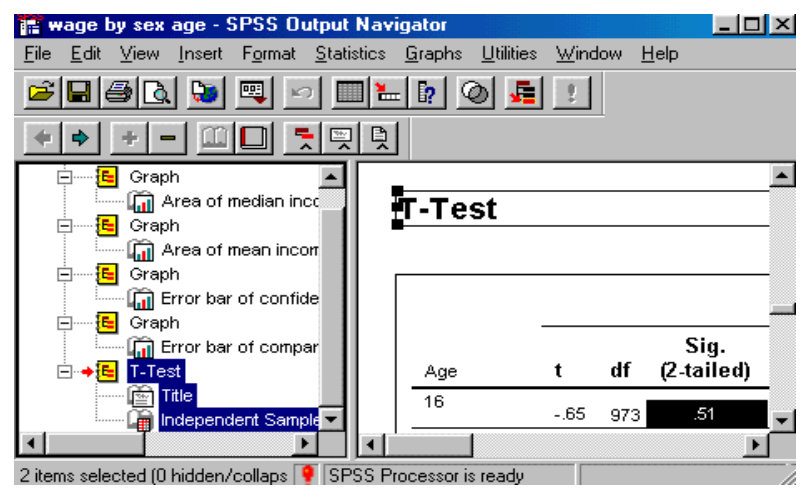

Lets open a Script file. Go to FILE/OPEN. In the area "Files of type" choose the file type "SPSSScript (.sbs)" as shown below. To locate the Script files, go to the folder "SPSS/Scripts/" on the path SPSS is installed163. (Or do a search for files ending with the extension ".sbs.")

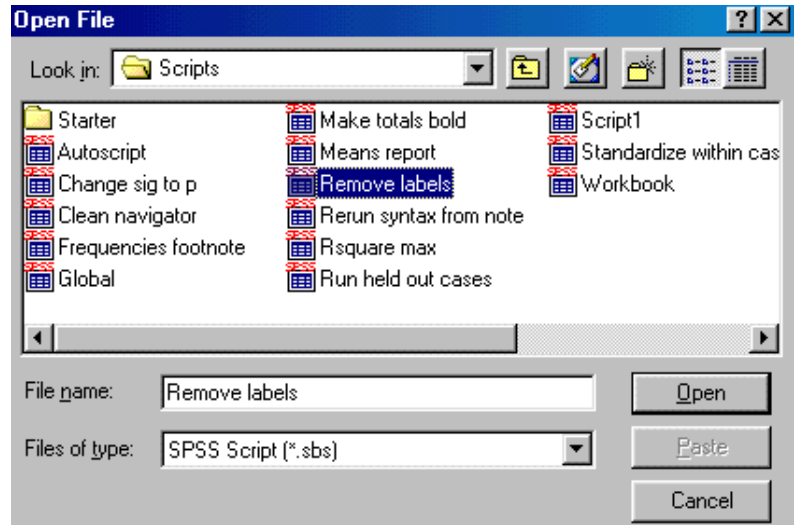

Click on "Open." The Script file opens in a new window called the "Script Editor" as shown inthe picture below.

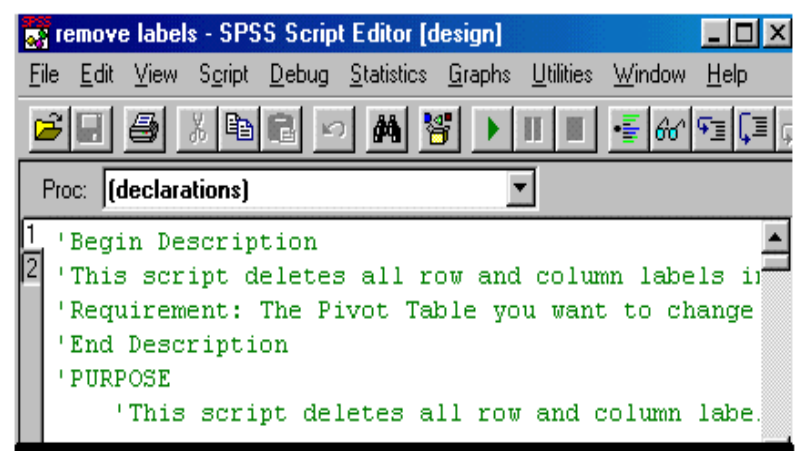

The Script file is basically a word-processing document in which text is written. The code starts with the line "'Begin Description." The lines of code that start with an apostrophe are comments that provide information to you on what the Script does and what requirements and actions it needs from you. In the next picture I show the entire description and purpose of the Script "Remove Labels."
Begin Description
This scrigr deletes all rou and colum labels is the selected Pivor Table. Pesuirenent: The Piwot Thole rou rant to change riat be selected.
End Description
potpost
This scrige deletes all ror and colan labels in the selected Pivor Table

Scroll down the page. You will see lines of text that do not have any apostrophe at the starting .These are the lines of functional code. Don't try to learn or worry about understanding the code. 


\section{Oation Explicit 'L11 variables wast be declarated before being usi}

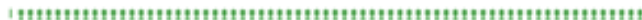

Swb tain

'Declare variables bere

Dis objpivottable ls Piwottable

Dis objIten Is ISpasiten:
Actually you don't need to change anything or do anything to the code. You just "Run" (that is,execute) it. To run the code, first see if the Script has any "Requirements" from the user. (Look at the lines that begin with an apostrophe-- one of them may start with the word "Requirements.")This Script requires that the user first choose one output table (also called "Pivot" table). So, first go to the "SPSS Output Navigator" window and choose one table. Then go back to the "Script Editor" window and click on the icon with a rightward-pointing arrow. The icon is shown in the next picture.

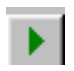

The code will run and perform the operations it is designed to do-- it will delete all row and column labels on all the tables in the open output window .Another way to run a script-- go to UTILITIES / RUN SCRIPT as shown in the picture below.

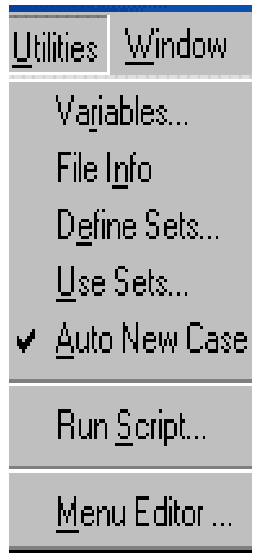

The available scripts will be shown in the "RUN SCRIPT" dialog box as shown in the next picture. When you click on the name of a script, its description will be shown under the box."Description" on the right-hand side. Within the description area, play close attention to the paragraph "Requirements." In this case, you are required to choose one output table (also called a"Pivot" table) and then run the script. So, first choose one table. Then, go to the Script window shown below and click on "RUN." The Script will be executed.

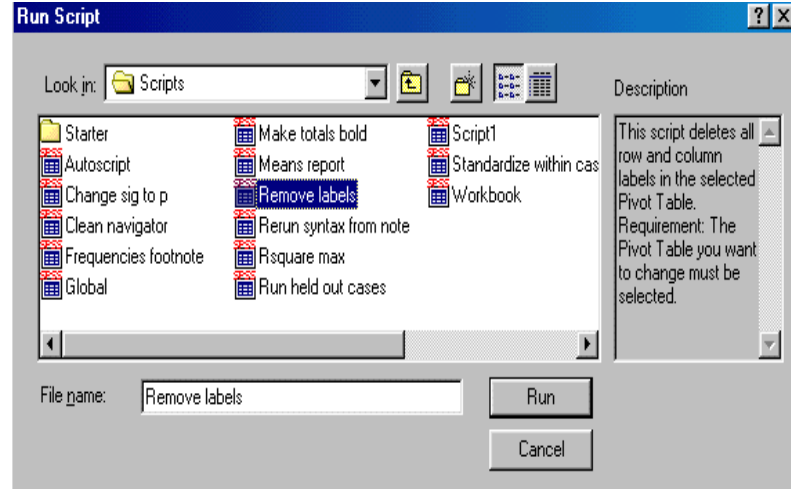

\section{USING SPSS SYNTAX}

The use of Syntax is best shown with an example. Please work through the example along with author. Open the sample data file provided in the file you downloaded .Go to GRAPHS / LINE GRAPH. Choose the option "Simple" and "Summary of individual cases." Click on

"Define." The following dialog box opens.

\begin{tabular}{|c|c|c|c|}
\hline \multirow{9}{*}{$\begin{array}{l}\text { gdp } \\
\text { gdp_1 } \\
\text { inv }\end{array}$} & \multirow{2}{*}{\multicolumn{2}{|c|}{ 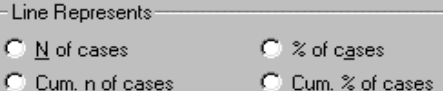 }} & OK \\
\hline & & & Paste \\
\hline & \multirow{2}{*}{\multicolumn{2}{|c|}{$\begin{array}{l}\text { (- Other summary function } \\
\qquad \begin{array}{l}\text { Variable: } \\
\text { MEAN [cons] }\end{array}\end{array}$}} & Reset \\
\hline & & & Cancel \\
\hline & \multicolumn{2}{|c|}{ Change Summary... } & Help \\
\hline & \multicolumn{2}{|l|}{$\begin{array}{l}\text { Category Axis: } \\
\text { Uear }\end{array}$} & \\
\hline & \multicolumn{2}{|c|}{$\begin{array}{l}\text { Template } \\
\Gamma \text { Use chart specifications from: }\end{array}$} & \\
\hline & \multicolumn{2}{|c|}{$\Gamma \underline{U}$ se chart specifications from: } & Iitles... \\
\hline & Eile... & & Qpptions... \\
\hline
\end{tabular}

Choose the options as shown above. Now, instead of clicking on the button "OK," click on the button "Paste." Doing this automatically launches the "Syntax Editor" window and automatically writes and pastes the code for the line graph you are planning to construct 165 . The syntax window is shown below.

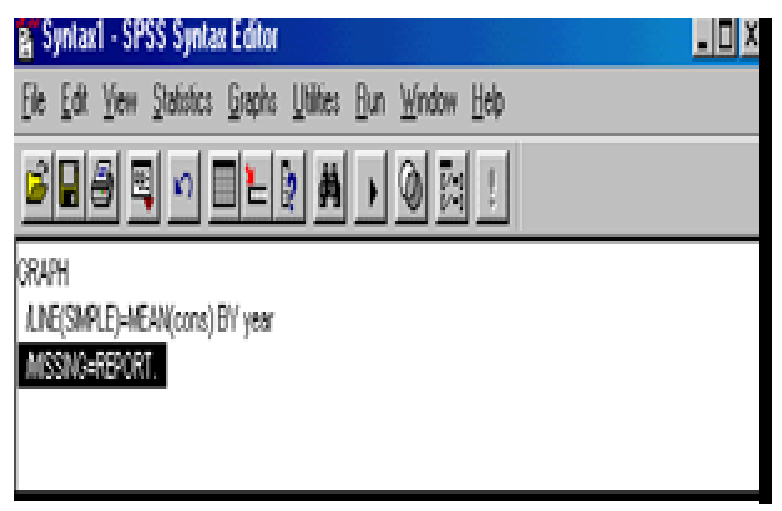

As shown above, the code for the graph is written on 3 lines. Just notice a few features:

- Variable names are in small case while all other code is in capital case

- Each line (apart from the first line) starts with the key "?."

- The bunch of code ends with a period. 
This is all you need to learn about the coding structure.

Two housekeeping steps to make your work more organized:

1. Place the cursor after the end of the code and hit "Enter" a few times. This provides empty space between bunches of code thereby increasing the ease of reading and using the code.(This is shown in the next picture.)

2. Save the Syntax file-- go to FILE/SAVE AS and save it as Syntax file (with the extension".sps.")

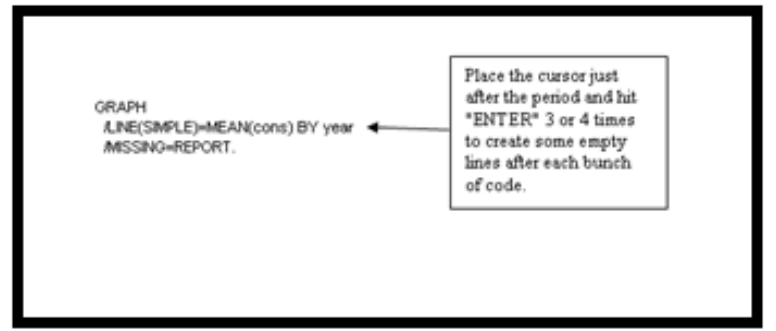

How does one use/execute the code? Using the mouse, highlight the 3 lines of code and go to RUN / ALL (or RUN / SELECTION) or click on the icon with the right-arrow sign (shown in the next picture.)

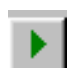

Let's do another example. Make the same line graph as in the previous example, but this time, in addition, click on the button "Titles" (within the dialog box for line graphs) and enter titles and footnotes as shown in the next picture.

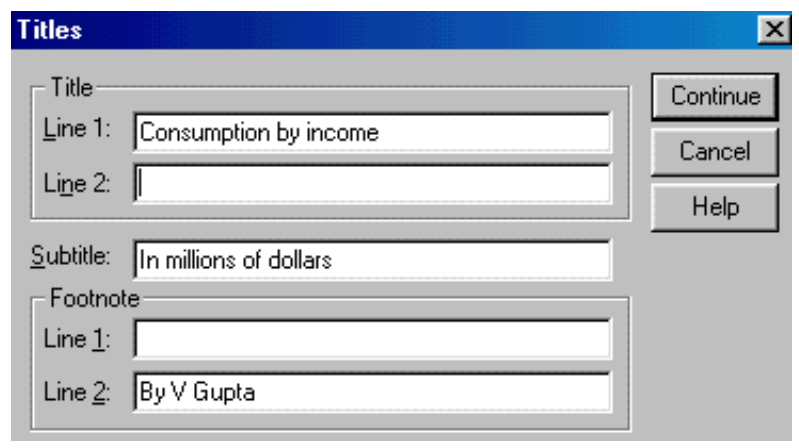

click on "Continue" and then on "Paste." The code is written and pasted onto the Syntax file automatically by SPSS. The code is shown in the next picture. (It is the second bunch of code.)

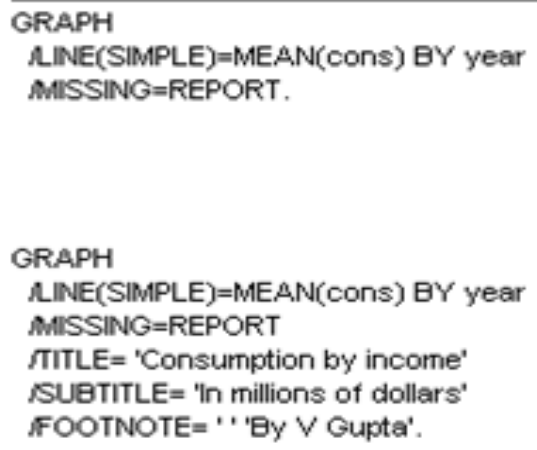

This time the code includes new lines that capture the titles and footnotes you wrote. To run the entire code (that is, both the line graphs) choose both bunches of code and go to RUN/

SELECTION. To run only one bunch of code (that is, only one of the line graphs) choose one bunch of code and go to RUN/SELECTION. (Do you now realize the importance of placing empty lines after each bunch of code?)After you paste every new bunch of code, go to FILE/SAVE. One more good housekeeping strategy-- writes some comments before each bunch of code. This comment may include what the code does, who pasted it, the date, etc. To write a comment, start

the line with an asterisk $\left(^{*}\right)$ and end the line with an asterisk and then a period $\left(^{*}\right.$.). This is shown in the next picture.

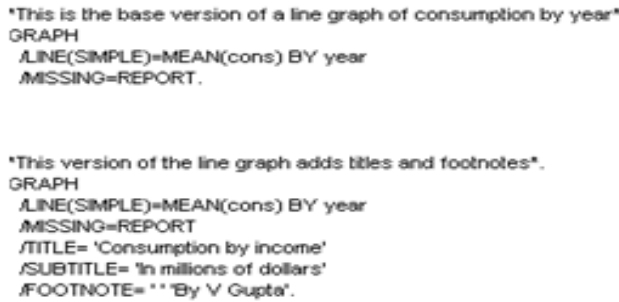

Continue writing code this way. Choose a procedure and click on the button "Paste" instead of" OK." And continue...

\subsection{A.Benifits Using Syntax}

Some major advantages of using Syntax are listed below:

1. Getting over any phobia/aversion to using (and maybe writing) software code. In general, becoming more confident with software technology.

2. Documenting all the work done in a project. If you use the simple point-and-click windows interface for your project then you will not have a record of all the procedures you conducted.

3. Because the Syntax allows you to document all your work, checking for errors becomes easier. With experience, you will be able to understand what the Syntax code says-then Checking for errors becomes very easy.

4. The main advantage is the massive saving of time and effort. How does syntax do this? Several ways, a few of which are listed below.

- Replication of code (including using Word to assist in replication) allows you to save considerable time (in using the point-and-click windows interface) as shown in the example above.

- Assume you want to run the same 40 procedures on 25 different files (say on data for five countries). If the files have the same variables that you are using in the procedures and the same variable names, then considerable time can be saved by creating the Syntax file using one country's data file and then running the same code on the data files of the other counties. In a follow-up chapter ('Advanced Programming in SPSS") I will show more ways to write time saving Syntax files.

- Assume you have several files with similar data but with different variable names. Create the syntax file for one data file. Then, for the other data files, just replace the

- Variable names on the original Syntax file! 
- A frustrating situation arises when you have to redo all you work because of data or other issues.166 SPSS can save an incredible amount of time as also the boredom produced by repeating tasks.

- After running some procedures, you may want to run them again on only a subset of the data file (see section 1.7 in my book), or separately for sub-groups of the data(see ch 10 in my book). Syntax makes this easy. If you have the syntax file for the procedures you conducted on the entire data file, then the same procedures can be redone for the sub-group(s) of the data by first making the sub-groups(s) and then rerunningthe code in the Syntax file.

\subsection{Using Word (or WordPerfect) to save time in creating code}

Microsoft Word has an extremely powerful "Find and Replace" facility. You can use this facility to save time in replicating SPSS code. In the earlier examples, I showed how to write code for a graph of "Consumption" and "Year." Let's use Word to replicate the code for graphs of" Investment" by "Year."Select the two bunches of code you "wrote" earlier. Go to EDIT / COPY. Open Microsoft Word(or WordPerfect). Go to EDIT / PASTE. The SPSS code is pasted onto the Word document as shown in the next picture.

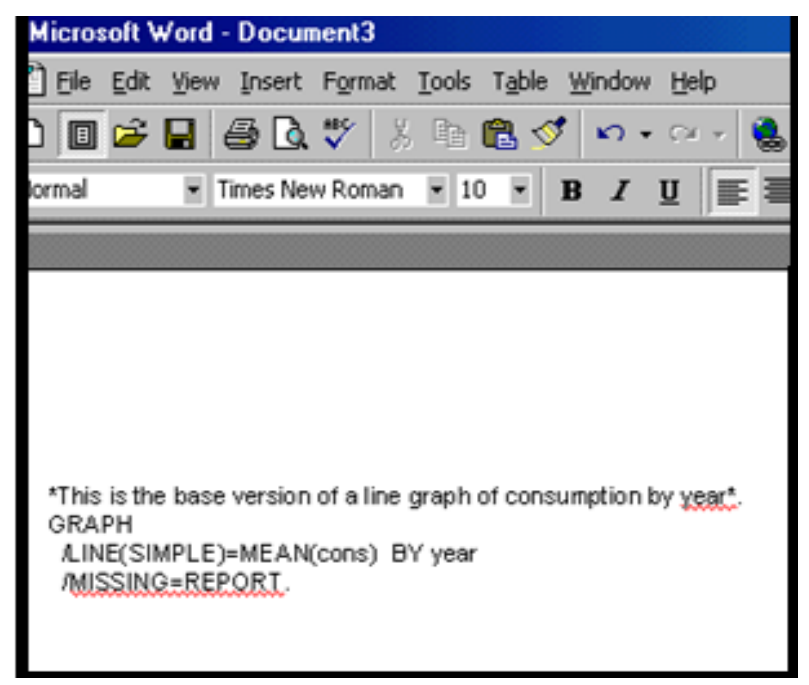

Go to EDIT / REPLACE and choose the options for replacing "cons" and "consumption" by "inv" and "investment," respectively. (See the next two pictures.)

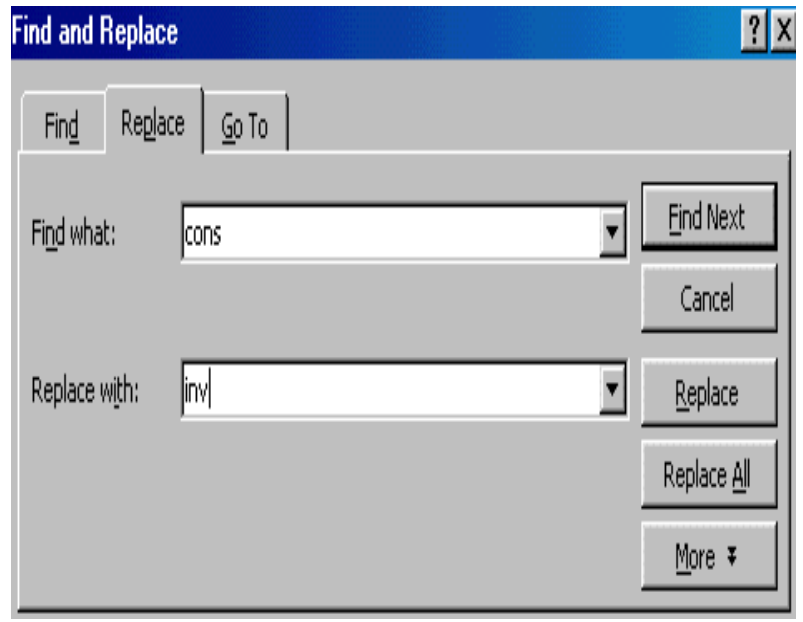

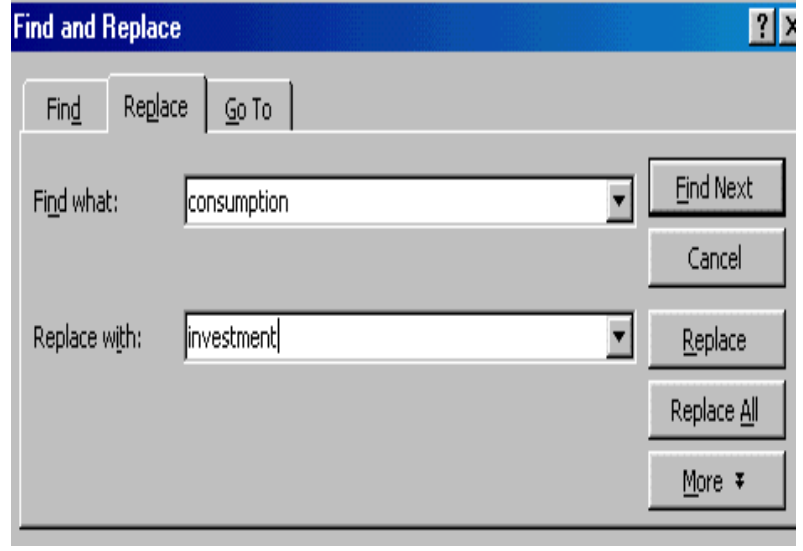

Copy the changed text (all of it) and go to EDIT / COPY. Go back to the SPSS syntax file and goto EDIT / PASTE. To run the two new bunches of code, choose the lines of code and go to RUN/ SELECTION.

\section{CONCLUSION}

Use of Syntax programming saves massive time and effort .For example Replication of code (including using Word to assist in replication) allows you to save considerable time. A frustrating situation arises when you have to redo all you work because of data or other issues.166 SPSS can save an incredible amount of time as also the boredom produced by repeating tasks. - After running some procedures, you may want to run them again on only a subset of the data file (see section 1.7 in my book), or separately for sub-groups of the data(see ch 10 in my book). Syntax makes this easy. If you have the syntax file for the procedures you conducted on the entire data file, then the same procedures can be redone for the sub-group(s) of the data by first making the sub-groups(s) and then rerunning the code in the Syntax file, but in case of scripting it does not happen hence syntax programming is better than that of Scripting Language .

\section{REFERENCES}

[1] SPSS Programming and Data Management, 2nd Edition. Raynald Levesque

[2] Ding ,SPSSa programming approach by Lora D. Delwiche and Susan J. Slaughter

[3] Business Research Methods and Statistics Using SPSS By RobertP Burns,Richard Burns

[4] SPSS: Stats Practically Short and Simple bySidney Tyrrell

[5] SPSS Web Books Regression with SPSSby Xiao Chen, Phil Ender, Michael Mitchell and Christine Wells .

[6] Discovering Statistics using IBM SPSS by ANDY FIELD.

[7] Using SPSS to Understand Research and Data Analysis Daniel Arkkelin Valparaiso University, daniel.arkkelin@valpo.edu

[8] IEEE paper The application of SPSS in empirical research of housing hedonic price by Xuyu Wang; School of Economics and Management, Tongji University, Shanghai, China . 